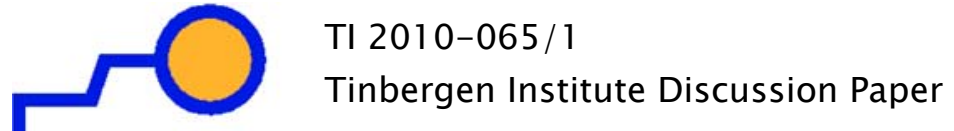 Axiomatization and Implementation of Discounted Shapley Values
}

\author{
René van den Brink ${ }^{7}$ \\ Yukihiko Funaki2
}

' VU University Amsterdam, and Tinbergen Institute;

2 School of Political Science and Economics, Waseda University, Tokyo. 


\section{Tinbergen Institute}

The Tinbergen Institute is the institute for economic research of the Erasmus Universiteit Rotterdam, Universiteit van Amsterdam, and Vrije Universiteit Amsterdam.

Tinbergen Institute Amsterdam

Roetersstraat 31

1018 WB Amsterdam

The Netherlands

Tel.: +31(0)205513500

Fax: $+31(0) 205513555$

Tinbergen Institute Rotterdam

Burg. Oudlaan 50

3062 PA Rotterdam

The Netherlands

Tel.: + $31(0) 104088900$

Fax: $+31(0) 104089031$

Most TI discussion papers can be downloaded at http://www.tinbergen.nl. 


\title{
Axiomatization and Implementation of Discounted Shapley Values
}

\author{
René van den Brink* Yukihiko Funaki ${ }^{\dagger}$
}

July 2, 2010

*Department of Econometrics and Tinbergen Institute, VU University, De Boelelaan 1105, 1081 HV Amsterdam, The Netherlands. E-mail: jrbrink@feweb.vu.nl

†Department of Economics, School of Political Science and Economics, Waseda University, 1-6-1 Nishi-Waseda, Shinjuku-Ku, Tokyo, 169-8050 Japan. E-mail: funaki@waseda.jp. Financial support from NWO-grant B 45-299 is gratefully ackonowledged. 


\begin{abstract}
We generalize the null player property (satisfied by the Shapley value) and nullifying player property (satisfied by the equal division solution) to the so-called $\delta$-reducing player property, stating that a $\delta$-reducing player (being a player such that any coalition containing this player earns a fraction $\delta \in[0,1]$ of the worth of that coalition without that player) earns a zero payoff. This property yields the null player property for $\delta=1$ and the nullifying player property for $\delta=0$. We show that efficiency, symmetry, linearity and this $\delta$-reducing player property characterizes the corresponding $\delta$-discounted Shapley value. Moreover, we provide a strategic implementation of these solutions where $\delta$ is a discount factor that determines the decrease in value to be distributed in the next round after the proposal is rejected and the remaining players (without the proposer) play a new round of bidding.
\end{abstract}

Keywords: Cooperative TU-game, Shapley value, equal division solution, $\delta$-discounted Shapley value, Axiomatization, Implementation, Discounting

JEL code: C71; C72

AMS subject classification: 91A12; 91A10 


\section{Introduction}

Recently, several solutions that make a trade-off between marginalism and egalitarianism in cooperative TU-games have been developed such as the egalitarian Shapley values, being convex combinations of the Shapley value and the equal division solution (see Joosten (1996)), and the (generalized) consensus values, being convex combinations of the Shapley value and the CIS-value (see Ju, Borm and Ruys (2007)) ${ }^{1}$. Although in all these solutions the Shapley value is the most marginalistic solution (see also Young (1985)), there are different possibilities with respect to the most egalitarian solution ${ }^{2}$. In van den Brink, Funaki and Ju (2007) it is shown that all egalitarian Shapley values have Sobolev's reduced game consistency (see Sobolev (1973)) in common, and differ only with respect to a standardness for two-player games. Such a standardness axiom requires that in a two-player game both players get a fraction of their singleton worth, and the remainder is split equally among them. The fraction of their singleton worth that the players can keep for themselves determines the weight put on the Shapley value.

Another comparison of the Shapley value and the equal division solution is made by van den Brink (2007) who considers several axiomatizations of the Shapley value and shows that replacing an axiom concerning null players (i.e. players whose marginal contribution to any coalition is zero) by a similar axiom concerning nullifying players (i.e. players whose presence in a coalition implies the coalition earns zero worth) characterizes the equal division solution. For example, in the original axiomatization of the Shapley value (see Shapley (1953)) by efficiency, the null player property, symmetry and additivity, replacing the null player property (which states that null players get a zero payoff) by the nullifying player property (which states that nullifying players get a zero payoff) characterizes the equal division solution ${ }^{3}$. In the underlying paper we want to make a trade-off between marginalism and egalitarianism by taking a combination of these two properties. In other words, we define a particular type of player to whom we assign a zero payoff. For $\delta \in[0,1]$ a player $i$ is called a $\delta$-reducing player in a game if any coalition containing this player earns a fraction $\delta$ of the worth of that coalition without player $i$. Then the $\delta$-reducing player property states that such a player gets a zero payoff. Clearly, $\delta=1$ implies that player $i$ 's marginal contributions are all zero, and thus yields the null player property of the Shapley value. On the other hand, $\delta=0$ implies that the worth of any coalition containing player $i$ is zero, and thus this yields the nullifying player property.

\footnotetext{
${ }^{1}$ The CIS-value, or equal surplus division solution, assigns to every TU-game the Center of the Imputation Set, i.e. every player gets its singleton worth and the remaining surplus is distributed equally among the players, and is discussed in Driessen and Funaki (1991).

${ }^{2}$ Different egalitarian solutions are compared in van den Brink and Funaki (2009).

${ }^{3} \mathrm{~A}$ similar result can be obtained for the CIS-value or equal surplus division solution.
} 
It turns out that replacing the null or nullifying player property (in the axiomatizations of the Shapley value, respectively, equal division solution) by this $\delta$-reducing player property yields the corresponding $\delta$-discounted Shapley value as considered in Joosten (1996) and Driessen and Radzik (2002). This class contains both the Shapley value and the equal division solution as extreme cases. However, for $\delta \in(0,1)$ this solution is not a convex combination of the Shapley value and the equal division solution, and thus this class is different from the class of egalitarian Shapley values. From Joosten (1996, Proposition 5.32) (see also Driessen and Radzik (2002)), it follows that all $\delta$-discounted Shapley values satisfy Hart and Mas-Colell's reduced game consistency (see Hart and Mas-Colell (1988, 1989)). In fact, he shows that for any $\delta \in[0,1]$ the corresponding solution is characterized by HM-consistency and the corresponding $\delta$-standardness for two player games.

Following Nash (1953), besides axiomatizations a second approach to characterize solutions is to find non-cooperative (strategic) implementations of these solutions. Our second characterization of the discounted Shapley values concerns such a strategic implementation. The bidding mechanism (extensive form game) which implements these solutions generalizes the one for the Shapley value given in Pérez-Castrillo and Wettstein (2001), differing only in the fact that we allow for discounting in the available surplus to be dirstibuted. Assuming that after each rejection of the proposal the amount available to be distributed among the remaining players in the next round is discounted by parameter $\delta \in[0,1]$, this modification of the mechanism of Pérez-Castrillo and Wettstein (2001) yields the corresponding $\delta$-discounted Shapley value as the payoff distribution in any subgame perfect equilibrium of this game.

The paper is organized as follows. Section 2 contains preliminaries on TU-games and solutions. In Section 3 we provide some axiomatic characterizations of the $\delta$-discounted Shapley values. Besides the original axiomatization of Shapley (1953) we also adapt the characterizations of Young (1985) and Chun (1992) for this class ${ }^{4}$. Section 4 provides a strategic implementation. Finally, Section 5 contains concluding remarks.

\section{Preliminaries}

A situation in which a finite set of players can obtain certain payoffs by cooperation can be described by a cooperative game with transferable utility, or simply a TU-game, being a pair $(N, v)$, where $N \subset \mathbb{N}$ is a finite set of players and $v: 2^{N} \rightarrow \mathbb{R}$ is a characteristic function on $N$ such that $v(\emptyset)=0$. For any coalition $S \subseteq N, v(S)$ is called the worth of coalition $S$. This is the transferable utility that the members of coalition $S$ can obtain by

\footnotetext{
${ }^{4}$ These already have been adapted for the equal division solution in van den Brink (2007).
} 
agreeing to cooperate. We denote the class of all TU-games by $\mathcal{G}$. A TU-game $(N, v)$ is monotone if $v(S) \leq v(T)$ whenever $S \subseteq T \subseteq N$. The unanimity game of coalition $T \subseteq N$, $T \neq \emptyset$, on $N$ is the game $\left(N, u_{T}\right)$ given by

$$
u_{T}(S)= \begin{cases}1 & \text { if } T \subseteq S \\ 0 & \text { otherwise }\end{cases}
$$

The standard game of coalition $T \subseteq N, T \neq \emptyset$, on $N$ is the game $\left(N, b_{T}\right)$ given by

$$
b_{T}(S)= \begin{cases}1 & \text { if } T=S \\ 0 & \text { otherwise }\end{cases}
$$

It is well-known that every game can be expressed as a unique linear combination of unanimity games as $v=\sum_{\substack{T \subseteq N \\ T \neq \emptyset}} \Delta_{v}(T) u_{T}$ with $\Delta_{v}(T)=\sum_{S \subseteq T}(-1)^{|T|-|S|} v(S)$ the Harsanyi dividends (see Harsanyi 1959)). Similar, every game can be expressed as a unique linear combination of standard games by $v=\sum_{\substack{T \subseteq N \\ T \neq \emptyset}} v(T) b_{T}$.

In the sequel we denote $n=|N|$ for the number of players in $N$. For generic coalitions $S, T \subseteq N$ we denote $s=|S|$ and $t=|T|$.

A payoff vector of game $(N, v)$ is an $n$-dimensional real vector $x \in \mathbb{R}^{n}$ which represents a distribution of the payoffs that can be earned by cooperation over the individual players. A (point-valued) solution for TU-games is a function $\psi$ which assigns a payoff vector $\psi(N, v) \in \mathbb{R}^{n}$ to every TU-game $(N, v) \in \mathcal{G}$ such that $\psi_{i}(\{i\}, v)=v(\{i\})$ for all $i \in \mathbb{N}$. Two well-known solutions are the Shapley value and the equal division solution. The Shapley value (Shapley (1953)) is the solution that assigns to every TU-game $(N, v)$ the payoff vector

$$
S h_{i}(N, v)=\sum_{S \subseteq N \backslash\{i\}} \frac{s !(n-s-1) !}{n !} m_{i}^{S}(v),
$$

where for every $i \in S$ and $S \subseteq N \backslash\{i\}$

$$
m_{i}^{S}(v)=v(S \cup\{i\})-v(S)
$$

is the marginal contribution of player $i$ to coalition $S$.

The equal division solution is the solution that distributes the worth $v(N)$ of the 'grand coalition' equally among all players and thus assigns to every TU-game $(N, v)$ the payoff vector

$$
E D_{i}(N, v)=\frac{v(N)}{n} \text { for all } i \in N .
$$


Joosten (1996, Chapter 5) introduces two classes of solutions with the Shapley value and equal division solution as extreme cases. The first is the class of egalitarian Shapley values and consists of all convex combinations of the Shapley value and the equal division solution ${ }^{5}$. Second, for $\delta \in[0,1]$, he introduces the solution $S h^{\delta}$ given by

$$
S h_{i}^{\delta}(N, v)=\sum_{S \subseteq N \backslash\{i\}} \frac{s !(n-s-1) !}{n !} \delta^{n-s-1}(v(S \cup\{i\})-\delta \cdot v(S)) \text { for all } i \in N .
$$

Later, these solutions have been called $\delta$-discounted Shapley values by Driessen and Radzik (2002). Joosten (1996) also showed that all $\delta$-discounted Shapley values satisfy Hart and Mas-Colell (HM) consistency. Even stonger, he showed that for any $\delta \in[0,1]$ the corresponding $\delta$-discounted Shapley value is characterized by HM-consistency and $\delta$ standardness for two player games, see Section 5 for details.

Example 2.1 Consider the 3-player unanimity games $\left(N, u_{\{2\}}\right)$ and $\left(N, u_{\{2,3\}}\right)$ on $N=$ $\{1,2,3\}$. In these games the payoffs of the players are given by $S h^{\delta}\left(N, u_{\{2\}}\right)=\left(\frac{2-\delta-\delta^{2}}{6}, \frac{1+\delta+\delta^{2}}{3}, \frac{2-\delta-\delta^{2}}{6}\right)$ and $\operatorname{Sh}^{\delta}\left(N, u_{\{2,3\}}\right)=\left(\frac{1-\delta}{3}, \frac{2+\delta}{6}, \frac{2+\delta}{6}\right)$. For example, taking $\delta=\frac{1}{2}$ we obtain $\operatorname{Sh}^{\frac{1}{2}}\left(N, u_{\{2\}}\right)=$ $\frac{1}{24}(5,14,5)$ and $S h^{\frac{1}{2}}\left(N, u_{\{2,3\}}\right)=\frac{1}{12}(2,5,5)$.

Next we recall some well-known properties of solutions for TU-games. First, we define two vectors associated to every player in a TU-game. The marginal vector $m^{i}(v) \in$ $\mathbb{R}^{2^{n-1}}$ of player $i$ is the vector of marginal contributions of player $i$ to coalitions $S \subseteq N \backslash\{i\}$, while the worth vector $v^{i} \in \mathbb{R}^{2^{n-1}}$ is the vector of worths of coalitions $v(S \cup\{i\}), S \subseteq N \backslash\{i\}$. A player $i \in N$ is a null player in $(N, v)$ if $m^{i}(v)$ is the zero vector with all components equal to zero, i.e. $v(S \cup\{i\})=v(S)$ for all $S \subseteq N \backslash\{i\}$. A player $i \in N$ is a nullifying player $^{6}$ in $(N, v)$ if $v^{i}$ is the zero vector, i.e. $v(S)=0$ for all $S \subseteq N$ with $i \in S$. A solution $\psi$

- satisfies the null player property if $\psi_{i}(N, v)=0$ whenever $i$ is a null player in $(N, v)$;

- satisfies the nullifying player property if $\psi_{i}(N, v)=0$ whenever $i$ is a nullifying player in $(N, v)$;

- is efficient ${ }^{7}$ if $\sum_{i \in N} \psi_{i}(N, v)=v(N)$ for all $(N, v) \in \mathcal{G}$;

- is linear if $\psi(N, \beta v+\gamma w)=\beta \psi(N, v)+\gamma \psi(N, w)$ for all $(N, v),(N, w) \in \mathcal{G}$ and $\beta, \gamma \in \mathbb{R}$, where $\beta v+\gamma w$ is given by $(\beta v+\gamma w)(S)=\beta v(S)+\gamma w(S)$ for all $S \subseteq N$;

\footnotetext{
${ }^{5}$ For every $\alpha \in[0,1]$, the $\alpha$-egalitarian Shapley value $\varphi^{\alpha}$ is the solution given by $\varphi^{\alpha}(N, v)=\alpha \operatorname{Sh}(N, v)+$ $(1-\alpha) E D(N, v)$.

${ }^{6}$ Deegan and Packel (1979) refer to nullifying players as zero players and use this property to characterize their (non-efficient) Deegan-Packel value.

${ }^{7}$ Efficient solutions are often called values.
} 
- is symmetric if $\psi_{i}(N, v)=\psi_{j}(N, v)$ for all $(N, v) \in \mathcal{G}$ and $i, j \in N$ such that $v(S \cup$ $\{i\})=v(S \cup\{j\})$ for all $S \subseteq N \backslash\{i, j\}$.

In the literature several axiomatizations of the Shapley value can be found. For example, Shapley (1953) characterizes the Shapley value by efficieny, symmetry, linearity and the null player property ${ }^{8}$. Other axiomatizations can be found in, e.g. Young (1985), Chun (1992) and van den Brink (2001). In van den Brink (2007) it is shown that replacing in these axiomatizations an axiom concerning null players (such as the null player property) by a similar axiom concerning nullifying players (such as the nullifying player property) characterizes the equal division solution.

\section{Reducing players: Axiomatization of $\delta$-discounted Shapley values}

We generalize the null- and nullifying player property as follows. For $\delta \in[0,1]$ we call player $i \in N$ a $\delta$-reducing player in game $(N, v)$ if $v(S \cup\{i\})=\delta v(S)$ for all $S \subseteq N \backslash\{i\}$. So, when player $i$ enters any coalition then the worth of this coalition is a fraction $\delta$ of the worth of the coalition without player $i$. Clearly, if $\delta=1$ this implies that the worth of no coalition changes when $i$ enters, i.e. a 1-reducing player is a null player. When $\delta=0$ then the worth of any coalition not containing $i$ becomes zero when $i$ enters, i.e. a 0 -reducing player is a nullifying player. Consequently the following property generalizes the null- as well as the nullifying player property.

- For $\delta \in[0,1]$, solution $\psi$ satisfies the $\delta$-reducing player property if $\psi_{i}(N, v)=0$ whenever $i$ is a $\delta$-reducing player in $(N, v)$.

Next, we show that this axiom together with efficiency, symmetry and linearity characterizes the corresponding $\delta$-discounted Shapley value for every $\delta \in[0,1]$. This follows similar as axiomatizations of the Shapley value and equal division solution, but using a different basis for TU-games. Consider a fixed player set $N$. For every $T \subseteq N, T \neq \emptyset$, and $\delta \in[0,1]$ we define the game $\left(N, d_{T}^{\delta}\right) \in \mathcal{G}$ by $d_{T}^{\delta}=\sum_{\substack{S \subseteq N \\ T \subseteq S}} \delta^{s-t} b_{S}$, and thus

$$
d_{T}^{\delta}(S)=\left\{\begin{array}{cl}
\delta^{s-t} & \text { if } T \subseteq S \\
0 & \text { otherwise }
\end{array}\right.
$$

Note that the game $d_{T}^{\delta}$ is the unanimity game $u_{T}$ of coalition $T \subseteq N$ if $\delta=1$, and it is the standard game $b_{T}$ of $T$ if $\delta=0$.

\footnotetext{
${ }^{8}$ This axiomatization is more often presented in this way although Shapley (1953) combines efficiency and the null player property into a carrier axiom.
} 
Lemma 3.1 For every $(N, v) \in \mathcal{G}$ and $\delta \in[0,1]$ there exist unique numbers $D_{v}^{\delta}(T) \in \mathbb{R}$ such that $v=\sum_{\substack{T \subseteq N \\ T \neq \emptyset}} D_{v}^{\delta}(T) d_{T}^{\delta}$.

\section{ProOF}

Consider a game $(N, v) \in \mathcal{G}$ and $\delta \in[0,1]$. Obviously, if $T=\{i\}$ then $D_{v}^{\delta}(\{i\})=v(\{i\})$ is uniquely determined since $d_{\{i\}}^{\delta}$ is the only game $d_{T}^{\delta}$ for which $d_{T}^{\delta}(\{i\}) \neq 0$. Proceeding by induction, suppose that the numbers $D_{v}^{\delta}\left(T^{\prime}\right)$ have been uniquely determined for any $T^{\prime} \subset N$ with $\left|T^{\prime}\right|<|T|$. Since the worth of any $S \subseteq N$ can be written as $v(S)=$ $\sum_{H \subseteq N} D_{v}^{\delta}(H) d_{H}^{\delta}(S)=\sum_{H \subseteq S} D_{v}^{\delta}(H) d_{H}^{\delta}(S)=D_{v}^{\delta}(S) d_{S}^{\delta}(S)+\sum_{H \subset S} D_{v}^{\delta}(H) d_{H}^{\delta}(S)$, it follows with the induction hypothesis and $d_{T}^{\delta}(T)=1$, that $D_{v}^{\delta}(T)=\frac{1}{d_{T}^{\delta}(T)}\left(v(T)-\sum_{H \subset T} D_{v}^{\delta}(H) d_{H}^{\delta}(T)\right)$ is uniquely determined.

Next, we can state our first main result, axiomatizing the $\delta$-discounted Shapley values ${ }^{9}$.

Theorem 3.2 Take $\delta \in[0,1]$. Solution $\psi$ satisfies efficiency, symmetry, linearity and the $\delta$-reducing player property if and only if $\psi=S h^{\delta}$.

\section{PROOF}

It is straightforward to verify that $S h^{\delta}$ satisfies these axioms. To show uniqueness suppose that solution $\psi$ satisfies the four axioms, and take $T \subset N, T \neq \emptyset$. We first show that every player $i \in N \backslash T$ is a $\delta$-reducing player in $\left(N, d_{T}^{\delta}\right)$. Consider $S \subseteq N \backslash\{i\}$. We distinguish two cases.

(i) Suppose that $T \not \subset S$. Then $T \not \subset S \cup\{i\}$, and thus $d_{T}^{\delta}(S \cup\{i\})=d_{T}^{\delta}(S)=0$, implying that $d_{T}^{\delta}(S \cup\{i\})=\delta d_{T}^{\delta}(S)$ for any $\delta \in[0,1]$.

(ii) Suppose that $T \subset S$. Then $T \subset S \cup\{i\}$, and thus $d_{T}^{\delta}(S)=\delta^{s-t}$ and $d_{T}^{\delta}(S \cup\{i\})=$ $\delta^{s+1-t}$, again implying that $d_{T}^{\delta}(S \cup\{i\})=\delta d_{T}^{\delta}(S)$.

Thus, $i \in N \backslash T$ is a $\delta$-reducing player in $\left(N, d_{T}^{\delta}\right)$.

(The remainder of the proof is similar as that in Shapley (1953), but using the basis $d_{T}^{\delta}$ instead of the unanimity basis ${ }^{10}$.) Consider any $(N, v) \in \mathcal{G}$ and $\delta \in[0,1]$. Efficiency and symmetry imply that $\psi_{i}\left(N, d_{N}^{\delta}\right)=\frac{1}{n}$ for all $i \in N$.

Now, let $T \subset N$. The $\delta$-reducing player property implies that $\psi_{i}\left(N, d_{T}^{\delta}\right)=0$ for all $i \in N \backslash T$. Efficiency then implies that $\sum_{i \in T} \psi_{i}\left(N, d_{T}^{\delta}\right)=\sum_{i \in N} \psi_{i}\left(N, d_{T}^{\delta}\right)=d_{T}^{\delta}(N)=\delta^{n-t}$. Thus, with symmetry it follows that $\psi_{i}\left(N, d_{T}^{\delta}\right)=\frac{\delta^{n-t}}{t}$ for all $i \in T$. Uniqueness for arbitrary

\footnotetext{
${ }^{9}$ We remark that the results in this section hold if we restrict ourselves to the class of all TU-games on a fixed player set $N$.

${ }^{10}$ Similar, van den Brink (2007) used the standard basis for characterizing the equal division solution.
} 
$(N, v) \in \mathcal{G}$ follows since linearity of $\psi$ and the fact that $v=\sum_{\substack{T \subseteq N \\ T \neq \emptyset}} D_{v}^{\delta}(T) d_{T}^{\delta}$ (see Lemma 3.1) imply that $\psi_{i}(N, v)=\sum_{\substack{T \subseteq N \\ T \neq \emptyset}} D_{v}^{\delta}(T) \psi_{i}\left(N, d_{T}^{\delta}\right)=\sum_{\substack{T \subseteq N \\ i \in T}} D_{v}^{\delta}(T) \frac{\delta^{n-t}}{t}$ for all $i \in N$.

Note that the $\delta$-reducing player property can be reformulated by saying that a player $i \in N$ gets a zero payoff if the convex combination $\delta m^{i}(v)+(1-\delta) v^{i}$ of the marginal vector $m^{i}(v) \in \mathbb{R}^{2^{n-1}}$ and the worth vector $v^{i} \in \mathbb{R}^{2^{n-1}}$ of player $i$ is the zero vector. So, instead of taking convex combinations of the Shapley value and the equal division solution (as in egalitarian Shapley values), taking convex combinations of the marginal vector (used in characterizing the Shapley value) and the worth vector (used in characterizing the equal division solution) in specifying a type of player that gets a zero payoff, yields a class of solutions that has the Shapley value and equal division solution as extreme cases, but are not convex combinations of these two solutions.

We want to stress the difference between the $\delta$-reducing player property and $\delta$ egalitarianism in Joosten (1996) or the quasi-dummy property in Ju, Borm and Ruys (2007). Whereas the latter two properties adapt the null player property by stating what a null player should get, with the $\delta$-reducing player property we specify a type of player that gets a zero payoff (like null players according to the null player property and nullifying players according to the nullifying player property). Of course, we could also characterize the class of $\delta$-discounted Shapley values $S h^{\delta}$ using a property similar to $\delta$-egalitarianism or the quasi-dummy property by specifying what is earned by null players. (The obvious proof is omitted.)

Proposition 3.3 If $i \in N$ is a null player in $(N, v) \in \mathcal{G}$ then

$$
S h_{i}^{\delta}(N, v)=(1-\delta) \sum_{S \subseteq N \backslash\{i\}} \delta^{n-s-1} \frac{s !(n-s-1) !}{n !} v(S) .
$$

Note that the $\delta$-discounted Shapley value $\operatorname{Sh}^{\delta}(N, v)$ can be rewritten as

$$
\begin{aligned}
S h_{i}^{\delta}(N, v)= & \sum_{\substack{S \subseteq N \backslash\{i\} \\
S \neq \emptyset}} \frac{s !(n-s-1) !}{n !} \delta^{n-s-1}(v(S \cup\{i\})-v(S))+ \\
& \sum_{\substack{S \subseteq N \backslash\{i\} \\
S \neq \emptyset}} \frac{s !(n-s-1) !}{n !} \delta^{n-s-1}(1-\delta) v(S) \text { for all } i \in N .
\end{aligned}
$$

From this it follows directly that all payoffs are nonnegative if the game is monotone.

Proposition 3.4 If $(N, v) \in \mathcal{G}$ is monotone then $\operatorname{Sh}_{i}^{\delta}(N, v) \geq 0$ for all $i \in N$.

We end this section by discussing adaptations of two well-known monotonicity axioms. For $\delta \in[0,1]$, solution $\psi$ 
- satisfies $\delta$-monotonicity if $\psi_{i}(N, v) \geq \psi_{i}(N, w)$ for every pair of games $(N, v),(N, w)$ and $i \in N$ such that $v(S \cup\{i\})-w(S \cup\{i\}) \geq \delta(v(S)-w(S))$ for all $S \subseteq N \backslash\{i\}$.

- satisfies coalitional $\delta$-equivalence if for every pair of games $(N, v),(N, w) \in \mathcal{G}$ it holds that $\psi_{i}(N, v+w)=\psi_{i}(N, v)$ whenever $i$ is a $\delta$-reducing player in $(N, w)$.

Taking $\delta=1$ in $\delta$-monotonicity yields strong monotonicity of Young (1985), while taking $\delta=1$ in coalitional $\delta$-equivalence yields coalitional strategic equivalence of Chun (1992). Taking $\delta=0$ in these two axioms yields coalitional monotonicity, respectively coalitional standard equivalence, see van den Brink (2007).

We can easily generalize the results of Young (1985) and Chun (1992). ${ }^{11}$

Theorem 3.5 Let $\delta \in[0,1]$. Then

(i) a solution $\psi$ satisfies efficiency, symmetry and $\delta$-monotonicity if and only if $\psi=S h^{\delta}$.

(ii) a solution $\psi$ satisfies efficiency, symmetry and coalitional $\delta$-equivalence if and only if $\psi=S h^{\delta}$.

\section{ProOF}

It is easy to verify that $S h^{\delta}$ satisfies efficiency, symmetry, $\delta$-monotonicity and coalitional $\delta$-equivalence. Since $\delta$-monotonicity implies coalitional $\delta$-equivalence, for uniqueness we only have to prove uniqueness in (ii). Therefore, suppose that solution $\psi$ satisfies the three axioms of (ii). Now, we show uniqueness by induction on $d(v)=\left|\left\{T \subseteq N \mid D_{v}^{\delta}(T) \neq 0\right\}\right|$ (in a similar way as in Young (1985) and Chun (1992) for the Shapley value and in van den Brink (2007) for the equal division solution).

If $d(v)=0$, then $(N, v)$ is a null game and efficiency and symmetry imply that $\psi_{i}(N, v)=0=S h_{i}^{\delta}(N, v)$ for all $i \in N$.

Proceeding by induction, assume that $\psi_{i}(N, w)=S h_{i}^{\delta}(N, w)$ if $d(w)<d(v)$. Let $H(v)=\left\{i \in N \mid D_{v}^{\delta}(T)=0\right.$ for all $\left.T \subseteq N \backslash\{i\}\right\}$. For every $i \in N \backslash H(v)$ there exists a $T \subseteq N \backslash\{i\}$ such that $D_{v}^{\delta}(T) \neq 0$. Coalitional $\delta$-equivalence and the induction hypothesis imply that $\psi_{i}(N, v)=\psi_{i}\left(N, v-D_{v}^{\delta}(T) d_{T}^{\delta}\right)=\operatorname{Sh}_{i}^{\delta}\left(N, v-D_{v}^{\delta}(T) d_{T}^{\delta}\right)=\operatorname{Sh}_{i}^{\delta}(N, v)$ for $i \in N \backslash H(v)$ and $T \subseteq N \backslash\{i\}$. With symmetry and efficiency it then follows that $\psi_{i}(N, v)=\frac{v(N)-\sum_{j \in N \backslash H(v)} S h_{j}^{\delta}(N, v)}{|H(v)|}=S h_{i}^{\delta}(N, v)$ for $i \in H(v)$.

\footnotetext{
${ }^{11}$ Also the axiomatization of van den Brink (2001) by efficiency, fairness and the null player property can be generalized, but the proof is more tedious and therefore deleted.
} 


\section{Implementation}

Before we give a strategic implementation of the solutions $S h^{\delta}$, we provide a recursive formula that generalizes that of Maschler and Owen (1989) for the Shapley value, which is given by

$$
S h_{i}(N, v)=\frac{v(N)-v(N \backslash\{i\})}{n}+\frac{1}{n}\left(\sum_{j \in N \backslash\{i\}} S_{i}(N \backslash\{j\}, v)\right) \text { for all } i \in N .
$$

Theorem 4.1 For every $\delta \in[0,1],(N, v) \in \mathcal{G}$ and $i \in N$, it holds that

$$
\operatorname{Sh}_{i}^{\delta}(N, v)=\frac{v(N)-\delta v(N \backslash\{i\})}{n}+\frac{\delta}{n}\left(\sum_{j \in N \backslash\{i\}} S h_{i}^{\delta}(N \backslash\{j\}, v)\right)
$$

\section{PROOF}

Take $\delta \in[0,1]$ and $(N, v) \in \mathcal{G}$. If $|N|=1$ then the statement of the theorem is obviously true since $S h_{i}^{\delta}(N, v)=v(\{i\})$ in that case. Proceeding by induction, suppose that the statement is true for all $\left(N^{\prime}, v\right) \in \mathcal{G}$ with $\left|N^{\prime}\right|<|N|$. It has been shown by Driessen and Radzik (2002) that the solutions $S h^{\delta}$ can be obtained by applying the Shapley value to the modified game $w^{\delta}$ given by $w^{\delta}(S)=\delta^{n-s} v(S), S \subseteq N$, i.e. for every $\delta \in[0,1]$ it holds that

$$
\operatorname{Sh}^{\delta}(N, v)=\operatorname{Sh}\left(N, w^{\delta}\right) .
$$

With (4.1) it then follows that

$$
\begin{aligned}
S h_{i}^{\delta}(N, v) & =S h_{i}\left(N, w^{\delta}\right) \\
& =\frac{w^{\delta}(N)-w^{\delta}(N \backslash\{i\})}{n}+\frac{\delta}{n}\left(\sum_{j \in N \backslash\{i\}} S h_{i}\left(N \backslash\{j\}, w^{\delta}\right)\right) \\
& =\frac{v(N)-\delta v(N \backslash\{i\})}{n}+\frac{\delta}{n}\left(\sum_{j \in N \backslash\{i\}} S h_{i}^{\delta}(N \backslash\{j\}, v)\right) .
\end{aligned}
$$

We obtain the recursive formula of Maschler and Owen (1989) by taking $\delta=1$. In proving the implementation later in this section we also use a modified balanced contributions property stating that for any game $(N, v)$, and for all $i, j \in N$ with $i \neq j$,

$$
S h_{i}^{\delta}(N, v)-\delta S h_{i}^{\delta}\left(N \backslash\{j\}, v_{N \backslash\{j\}}\right)=S h_{j}^{\delta}(N, v)-\delta S h_{j}^{\delta}\left(N \backslash\{i\}, v_{N \backslash\{i\}}\right)
$$


This follows straightforward from (4.2) and the balanced contributions property of Myerson (1980), which is obtained by taking $\delta=1 .^{12}$

Next, we provide a non-cooperative (strategic) implementation of the $\delta$-discounted Shapley values. In the literature various implementations of the Shapley value can be found, see e.g. Gul (1989), Hart and Mas-Colell (1996) and Pérez-Castrillo and Wettstein (2001). In this paper we modify the bidding mechanism of Pérez-Castrillo and Wettstein (2001). This bidding mechanism proceeds in rounds, where each round consists of four stages. In the first stage of round 1, all players make bids to each other and the player with the highest net bid (being the difference between the sum of all bids made to the other players and the sum of all bids received from the other players) is chosen to be the proposer in stage 2 (where ties are broken by assigning each of the players with highest net bid to be the proposer with equal probability). The 'winner' of this bidding pays the other players the bids that it offered. In the second stage the proposer makes a proposal (i.e. proposes a payoff) to every other player. In stage 3 the other players, sequentially, accept or reject the proposal. The proposal is accepted if all other players accept, and is rejected if at least one other player rejects it. In stage 4 , if the proposal is accepted the players are paid the proposed payoffs (additional to the bids made by the proposer in stage 1), and if the proposal is rejected then the proposer leaves the game and receives its own singleton worth (from which it has to pay the bids made in stage 1) while the other players go to the next round to bid and bargain over their worth $v\left(N \backslash \alpha_{1}\right)$, where $\alpha_{1}$ is the proposer in the first round.

This second round has the same four stages as the first round (but with $n-1$ players). The game proceeds untill at some round all remaining players accept the offer by the proposer or, after a sequence of rejections, round $n$ is reached in which there is only one player who just gets its singleton worth (plus all the net bids it received in all the first stages of all rounds). Pérez-Castrillo and Wettstein (2001) showed that, if the cooperative TU-game $(N, v)$ is zero-monotonic, then the payoffs in any subgame perfect equilibrium of this extensive form game are equal to the Shapley value of $(N, v)$.

In this bidding mechanism there is no discounting. After rejection of the proposal in the first round, in the next round the remaining players bid for the worth $v\left(N \backslash i_{1}^{*}\right)$, and so on. In this section we modify this bidding mechanism by discounting the worths to be distributed each time a proposal is rejected and the players move to a next round, by factor $\delta \in[0,1]$.

\footnotetext{
${ }^{12} \mathrm{By}$ the Shapley value satisfying the balanced contributions property of Myerson (1980), it follows that $S h_{i}^{\delta}(N, v)-\delta S h_{i}^{\delta}\left(N \backslash\{j\}, v_{N \backslash\{j\}}\right)=S h_{i}\left(N, w^{\delta}\right)-\delta S h_{i}\left(N \backslash\{j\}, \delta^{-1} w_{N \backslash\{j\}}^{\delta}\right)=S h_{j}\left(N, w^{\delta}\right)-S h_{j}(N \backslash$ $\left.\{i\}, w_{N \backslash\{i\}}^{\delta}\right)=S h_{j}\left(N, w^{\delta}\right)-S h_{j}\left(N \backslash\{i\}, w_{N \backslash\{i\}}^{\delta}\right)=S h_{j}^{\delta}(N, v)-\delta S h_{j}^{\delta}\left(N \backslash\{i\}, v_{N \backslash\{i\}}\right)$, where the first equality follows since $w_{N \backslash\{i\}}^{\delta}(S)=\delta^{n-1-s} v(S)=\delta^{-1} w^{\delta}(S)$ for all $S \subseteq N \backslash\{i\}$.
} 
To formally describe the bidding mechanism, let $N_{t}$ be the player set of the game with which each round $t \in\{1, \ldots, n\}$ will start, so $N_{1}=N$. For $T \subset N$ the restricted game $\left(T, v_{T}\right) \in \mathcal{G}$ is given by $v_{T}(S)=v(S)$ for all $S \subseteq T$.

\section{The bidding mechanism}

Round $t, t \in\{1, \ldots, n-1\}$ :

Stage 1: Each player $i \in N_{t}$ makes bids $b_{j}^{i} \in \mathbb{R}$ for every $j \neq i$. For each $i \in N_{t}$, let $B^{i}=\sum_{j \in N_{t} \backslash\{i\}}\left(b_{j}^{i}-b_{i}^{j}\right)$, be the net bid of player $i$. Let $\alpha_{t}$ be the player with the highest net bid of round $t$. (In case of a non-unique maximizer we choose any of these maximal bidders to be the 'winner' with equal probability.) Once $\alpha_{t}$ has been chosen, player $\alpha_{t}$ pays every other player $j \in N_{t} \backslash\left\{\alpha_{t}\right\}$, its offered bid $b_{j}^{\alpha_{t}}$. Player $\alpha_{t}$ becomes the proposer in the next stage. Go to Stage 2.

Stage 2: Player $\alpha_{t}$ proposes an offer $y_{j}^{\alpha_{t}} \in \mathbb{R}$ to every player $j \in N_{t} \backslash\left\{\alpha_{t}\right\}$. (This offer is additional to the bids paid at stage 1.) Go to Stage 3.

Stage 3: The players other than $\alpha_{t}$, sequentially, either accept or reject the offer. If at least one player rejects it, then the offer is rejected. Otherwise, the offer is accepted. Go to Stage 4.

Stage 4: If the offer is accepted, then each player $j \in N_{t} \backslash\left\{\alpha_{t}\right\}$ receives $y_{j}^{\alpha_{t}}$ and player $\alpha_{t}$ obtains the remainder $\delta^{t-1} v\left(N_{t}\right)-\sum_{j \in N_{t} \backslash\left\{\alpha_{t}\right\}} y_{j}^{\alpha_{t}}$ of the discounted payoff at this stage in Round $t$. Hence, in this case the final payoff to player $j \in N_{t} \backslash\left\{\alpha_{t}\right\}$ is $y_{j}^{\alpha_{t}}+b_{j}^{\alpha_{t}}+\sum_{k=1}^{t-1} b_{j}^{\alpha_{k}}$, while player $\alpha_{t}$ receives $\delta^{t-1} v\left(N_{t}\right)-\sum_{j \in N_{t} \backslash\left\{\alpha_{t}\right\}}\left(y_{j}^{\alpha_{t}}+\right.$ $\left.b_{j}^{\alpha_{t}}\right)+\sum_{k=1}^{t-1} b_{\alpha_{t}}^{\alpha_{k}}$. Stop.

If the offer is rejected then player $\alpha_{t}$ leaves the game and obtains its standalone payoff $\delta^{t-1} v\left(\left\{\alpha_{t}\right\}\right)$, while the players in $N_{t} \backslash\left\{\alpha_{t}\right\}$ proceed to round $t+1$ to bargain over $\delta^{t} v\left(N_{t} \backslash\left\{\alpha_{t}\right\}\right)$.

Round $n: N_{n}=N_{n-1} \backslash\left\{\alpha_{n-1}\right\}$. Since $N_{n}$ is a singleton coalition it is a one-player (sub)game in this round. The game immediately stops such that player $i \in N_{n}$ gets its discounted stand-alone payoff $\delta^{n-1} v\left(N_{n}\right)$. Its final payoff thus is $\delta^{n-1} v\left(N_{n}\right)+$ $\sum_{k=1}^{n-1} b_{i}^{\alpha_{k}}$.

As mentioned above, Pérez-Castrillo and Wettstein (2001) showed that for $\delta=1$ this bidding mechanism implements the Shapley value for zero-monotonic games. It turns out that this mechanism with discounting implements the corresponding $\delta$-discounted Shapley value as subgame perfect equilibrium (SPE) outcome if the original TU-game is $\delta$-monotonic 
meaning that $v(S) \geq \delta v(S \backslash\{i\})+v(\{i\})$ for all $S \subseteq N$ and all $i \in S .{ }^{13}$ Zero-monotonicity then is obtained by taking $\delta=1$. Note that for nonnegative games (i.e. $v(S) \geq 0$ for all $S \subseteq N), \delta$-monotonicity is weaker than zero-monotonicity for every $\delta \in(0,1]^{14}$ and thus also weaker than superadditivity. Thus, the implementation also holds if we just require the game to be nonnegative and zero-monotonic for all $\delta \in[0,1]$.

Theorem 4.2 Let $\delta \in[0,1]$, and let $(N, v) \in \mathcal{G}$ be a $\delta$-monotonic game. Then the outcome in any subgame perfect equilibrium of the bidding mechanism coincides with the payoff vector $\operatorname{Sh}^{\delta}(N, v)$.

\section{ProOF}

The proof follows the lines of the proof of the implementation of the Shapley value in Pérez-Castrillo and Wettstein (2001) (Theorem 1), and therefore we only mention the differences. First, Pérez-Castrillo and Wettstein (2001) construct a strategy profile that is an SPE in their bidding game and yields the Shapley value payoffs as outcomes. In this strategy profile, in all actions of player $i$ we discount the part that is based on payoffs in restricted games on the set of players without the proposer $\alpha_{t}$ (in the bidding in stage 1 , the proposals in stage 2 if $i$ is the proposer, and what proposals $i$ will accept in stage 3 if $i$ is not the proposer), i.e. in round $t \in\{1, \ldots, n-1\}$ we just replace $\phi_{j}\left(N_{t} \backslash\{i\}\right)$ by $\delta \phi_{j}\left(N_{t} \backslash\{i\}\right)$ and replace $\phi_{i}\left(N_{t} \backslash\{j\}\right)$ by $\delta \phi_{i}\left(N_{t} \backslash\{j\}\right) .{ }^{15}$ All net bids being zero can be shown using the modified balanced contributions (4.3) instead of balanced contributions.

Checking that the corresponding strategies yield an SPE goes similar as in PérezCastrillo and Wettstein (2001), but under the condition of $\delta$-monotonicity.

Then, Pérez-Castrillo and Wettstein (2001) show that any SPE yields the Shapley value payoffs by a series of claims. Claim (a) states that in any SPE all players $i \neq \alpha_{t}$ that are not proposer in round $t \in\{1, \ldots, n-1\}$ accept the offer of proposer $\alpha_{t}$ if that is greater than their payoff $\phi_{i}\left(N_{t} \backslash\left\{\alpha_{t}\right\}\right)$, and the offer is rejected if at least one of those players $i \neq \alpha_{t}$ gets an offer smaller than $\phi_{i}\left(N_{t} \backslash\left\{\alpha_{t}\right\}\right)$. We just discount the payoffs on the player set $N_{t} \backslash\left\{\alpha_{t}\right\}$ by multiplying these payoffs by $\delta$, i.e. replacing $\phi_{i}\left(N_{t} \backslash\left\{\alpha_{t}\right\}\right)$ by $\delta \phi_{i}\left(N_{t} \backslash\left\{\alpha_{t}\right\}\right)$.

Claim (b) states that if $v\left(N_{t}\right)>v\left(N_{t} \backslash\left\{\alpha_{t}\right\}\right)+v\left(\left\{\alpha_{t}\right\}\right)$, the only SPE of the (sub)game that starts at stage 2 is such that the proposer offers $\phi_{i}\left(N_{t} \backslash\left\{\alpha_{t}\right\}\right)$ to all $i \neq \alpha_{t}$, and at

\footnotetext{
${ }^{13}$ We remark that under the alternative condition $v(S) \geq \delta(v(S \backslash\{i\})+v(\{i\}))$ in stage 4 of the bidding mechanism we could allow proposer $\alpha_{t}$ in round $t$ to obtain $\delta^{t} v\left(\alpha_{t}\right)$ if its proposal is rejected.

${ }^{14}$ For any pair of discount factors $\delta, \delta^{\prime} \in[0,1]$ with $\delta<\delta^{\prime}$, a nonnegative game is $\delta$-monotonic if it is $\delta^{\prime}$-monotonic.

${ }^{15}$ Pérez-Castrillo and Wettstein (2001) shorten payoffs $\phi_{i}\left(T, v_{T}\right)$ by $\phi_{i}(T)$ for $T \subset N$. Also, they use the notation $N$ for the player set in any subgame, while in our notation $N$ refers to the full player set, and subgames are played on subsets $N_{t}$ in round $t \in\{1, \ldots, n-1\}$.
} 
stage 3 every player $i \neq \alpha_{t}$ accepts any offer greater or equal than $\phi_{i}\left(N_{t} \backslash\left\{\alpha_{t}\right\}\right)$ and rejects all smaller offers. Again we simply discount the payoffs of restricted games and replace $\phi_{i}\left(N_{t} \backslash\left\{\alpha_{t}\right\}\right)$ by $\delta \phi_{i}\left(N_{t} \backslash\left\{\alpha_{t}\right\}\right)$. Using $\delta$-monotonicity, this claim is shown if $v\left(N_{t}\right)>\delta v\left(N_{t} \backslash\left\{\alpha_{t}\right\}\right)+v\left(\left\{\alpha_{t}\right\}\right)$, i.e. we also discount the surplus $v\left(N_{t} \backslash\left\{\alpha_{t}\right\}\right)$. (Also in the proof of this claim everywhere we replace $v\left(N_{t} \backslash\left\{\alpha_{t}\right\}\right)$ by $\delta v\left(N_{t} \backslash\left\{\alpha_{t}\right\}\right)$.

Claim (c) of Pérez-Castrillo and Wettstein (2001) states that in any SPE, the net bids of all players are equal, and therefore equal to zero. For this claim discounting is not relevant, and for our mechanism this is proved in the same way ${ }^{16}$.

Claim (d) of Pérez-Castrillo and Wettstein (2001) states that in any SPE, each player's payoff is the same regardless who is chosen as the proposer. Also the proof of this claim does not need discounting, and is identical for all discounted Shapley values.

Finally Claim (e), stating that in any SPE the final payoff received by each of the players coincides with their Shapley value, uses Maschler and Owen (1989)'s recursive formula for the Shapley value (see (4.1)). Discounting the payoff $v\left(N_{t} \backslash\{i\}\right)$ by $\delta v\left(N_{t} \backslash\{i\}\right)$, we can apply Theorem 4.1 to obtain a similar result for any discounted Shapley value.

This completes the proof ${ }^{17}$.

In the bidding mechanism above we presented the parameter $\delta$ as a discount factor which determines the discounting of the available worth going from one round of negotiation to the next after a rejection of the proposal. Alternatively, the parameter $\delta$ can be interpreted as a probability of breakdown of the negotiations after a rejection. In van den Brink, Funaki and Ju (2007) the mechanism of Pérez-Castrillo and Wettstein (2001) is adapted to obtain a two-level bidding mechanism implementing any egalitarian Shapley value, i.e. any convex combination of the Shapley value and equal division solution. This modification concerns a possibility of breakdown of the negotiations after rejection of the proposal only in the first round of the negotiations. After that the mechanism is the same as that of Pérez-Castrillo and Wettstein (2001), so there is no possibility of breakdown anymore. This kind of breakdown may occur, for example, when the players do not know each other and speak different languages. Then it might be that after rejection in the first round they decide that they cannot communicate and bargain with each other. But if they agree that they can bargain together then they will bargain untill they reach an agreement or only one player is left.

\footnotetext{
${ }^{16}$ In the proof of this claim in Pérez-Castrillo and Wettstein (2001) the player $j \notin \Omega$ should be one with the highest net bid among those not in $\Omega$, where $\Omega$ is the set of players in $N$ with the highest net bid.

${ }^{17} \mathrm{~A}$ fully detailed proof can be obtained from the authors on request.
} 


\section{Concluding remarks}

In this paper we have characterized the $\delta$-discounted Shapley values by replacing the null-, respectively, nullifying player property that are used in characterizations of the Shapley value, respectively, the equal division solution, by the $\delta$-reducing player property (with the null- and nullifying player properties as special cases).

As a second characterization, we modified the bidding mechanism of Pérez-Castrillo and Wettstein (2001) implementing the Shapley value, by allowing discounting of the worths to be distributed after every rejection of the proposal with a uniform discount factor $\delta$, so that the payoffs in all subgame perfect equilibiria of the bidding game coincide with the corresponding $\delta$-discounted Shapley value.

We already mentioned in Section 2 that Joosten (1996) showed that for any $\delta \in[0,1]$ the corresponding $\delta$-discounted Shapley value is characterized by Hart and Mas-Colell consistency and $\delta$-standardness for two player games. To be precize, a solution $\psi$

- satisfies HM-consistency if $\psi_{i}(N, v)=\psi_{i}\left(T, \bar{v}_{T}^{\psi}\right)$ for every $(N, v) \in \mathcal{G}, T \subseteq N$, and $i \in T$, where the reduced game $\left(T, \bar{v}_{T}^{\psi}\right)$ is given by $\bar{v}_{T}^{\psi}(S)=v\left(S \cup T^{c}\right)-\sum_{j \in T^{c}} \psi_{j}(S \cup$ $\left.T^{c}, v_{S \cup T^{c}}\right)$ for all $S \subseteq T$ with $T^{c}=N \backslash T$.

- satisfies $\delta$-standardness for two-player games, $\delta \in[0,1]$, if for every $(N, v) \in \mathcal{G}$ with $N=\{i, j\}, i \neq j$, it holds that $\psi_{i}(N, v)=\delta v(\{i\})+\frac{1}{2}(v(N)-\delta(v(\{i\})+v(\{j\})))$.

Specific choices of $\delta \in[0,1]$ give different versions of standardness for two-player games as encountered in the literature. Taking $\delta=1$ yields standardness for two-player games as considered in, e.g. Hart and Mas-Colell $(1988,1989): \psi_{i}(N, v)=v(\{i\})+$ $\frac{1}{2}(v(N)-v(\{i\})-v(\{j\}))$ with $N=\{i, j\}$. Taking $\delta=0$, yields egalitarian standardness for two-player games: $\psi_{i}(N, v)=\frac{1}{2} v(N)$ for $i \in N$. As a corollary from Joosten (1996, Proposition 5.32), and Theorem 3.2 we thus obtain that a solution satisfies efficiency, symmetry, linearity and the $\delta$-reducing player property if and only if it satisfies HMconsistency and $\delta$-standardness for two-player games.

To compare, in van den Brink, Ju and Funaki (2007) it is shown that each egalitarian Shapley value (i.e. convex combination of the Shapley value and equal division solution) satisfies Sobolev (1973)'s reduced game consistency. In fact, they show that each egalitarian Shapley value is characterized by Sobolev's consistency and the corresponding $\delta$-standardness for two-player games.

At the end of the previous section we already remarked that the egalitarian Shapley values can be implemented by applying discounting (or possibility of breakdown of the negotiations) only in the first round, while in this paper it is shown that the discounted Shapley values are implemented by applying discounting in every period. One plan for 
future research is to consider what classes of solutions are obtained by a strategic implementation but where discounting is applied only in the first $k \in\{2, \ldots n-1\}$ rounds.

We end by considering the duals of the $\delta$-discounted Shapley values. The dual game of game $(N, v)$ is the game $\left(N, v^{*}\right)$ given by $v^{*}(N)=v(N)-v(N \backslash S)$ for all $S \subseteq N$. Instead of looking what coalition $S$ can earn, the dual of a game considers what the 'grand coalition' $N$ looses when coalition $S$ does not cooperate with the other players anymore. The dual of a solution $\psi$ is the solution $\psi^{*}(N, v)=\psi\left(N, v^{*}\right)$ for all $(N, v) \in \mathcal{G}$. A solution $\psi$ is self-dual if $\psi^{*}(N, v)=\psi(N, v)$ for all $(N, v) \in \mathcal{G}$. It is known that the Shapley value and the equal division solution are self-dual. For $\delta \in[0,1]$, denoting $\widehat{w^{\delta}}(S)=\delta^{n-s} v^{*}(S)=\delta^{n-s}(v(N)-v(N \backslash S))$ for all $S \subseteq N$, its dual game is ${\widehat{w^{\delta}}}^{*}(S)=$ $\widehat{w^{\delta}}(N)-\widehat{w^{\delta}}(N \backslash S)=\delta^{n-n} v(N)-\delta^{n-n+s}(v(N)-v(S))=\left(1-\delta^{s}\right) v(N)+\delta^{s} v(S)$. Denoting $\bar{v}(S)=\left(1-\delta^{s}\right) v(N)$ and $\widetilde{v}(S)=\delta^{s} v(S)$ for all $S \subseteq N$, we have

$$
\begin{aligned}
& S h_{i}^{\delta^{*}}(N, v)=S h_{i}^{\delta}\left(N, v^{*}\right)=S h_{i}\left(N, \widehat{w^{\delta}}\right)=S h_{i}(N, \bar{v})+S h_{i}(N, \widetilde{v}) \\
& =\frac{1-\delta^{n}}{n} v(N)+\sum_{S \subseteq N \backslash\{i\}} \frac{s !(n-s-1) !}{n !}\left(\delta^{s+1} v(S \cup\{i\})-\delta^{s} v(S)\right) \\
& =\frac{1-\delta^{n}}{n} v(N)+\sum_{S \subseteq N \backslash\{i\}} \frac{s !(n-s-1) !}{n !} \delta^{s}(\delta v(S \cup\{i\})-v(S)) \text { for all } i \in N .
\end{aligned}
$$

Studying the dual class of $\delta$-discounted Shapley values is also a plan for future research.

\section{References}

Brink, R. van den (2001), "An Axiomatization of the Shapley Value using a Fairness Property", International Journal of Game Theory, 30, 309-319.

Brink, R. van den (2007), "Null or Nullifying Players: the Difference between the Shapley Value and Equal Division Solutions", Journal of Economic Theory, 136, 767-775.

Brink, R. van den and Y. Funaki (2009) "Axiomatizations of a Class of Equal Surplus Sharing Solutions for TU-Games", Theory and Decision, 67, 303-340.

Brink, R. van den, Y. Funaki, and Y. Ju (2007), "Consistency, Monotonicity and Implementation of Egalitarian Shapley Values", Tinbergen Discussion Paper 07/062-1, Tinbergen Institute and Free University, Amsterdam.

Driessen, T.S.H., and Y. Funaki (1991) "Coincidence of and Collinearity between Game Theoretic Solutions", OR Spektrum, 13, 15-30. 
Driessen, T.S.H., and T. Radzik (2002), "A Weighted Pseudo-potential Approach to Values for TU games", International Transactions in Operational Research (ITOR), 9, $1-18$.

Gul, F. (1989) "Bargaining Foundations of Shapely Value", Econometrica, 57, 81-95.

Harsanyi, J.C. (1959), "A Bargaining Model for Cooperative N-person Games", in Contributions to the Theory of Games IV (A.W. Tucker and R.D. Luce, Eds.), pp. 325-355. Princeton: Princeton UP.

Hart, S., and A. Mas-Colell (1988), "The Potential of the Shapley Value", in: The Shapley Value. Essays in Honor of L.S. Shapley (ed. A.E. Roth), Cambridge University Press, pp. 127-137.

Hart, S., and A. Mas-Colell , (1989), "Potential, Value and Consistency", Econometrica, $57,589-614$.

Hart, S., and A. Mas-Colell , (1996), "Bargaining and Value", Econometrica, 64, 357-380.

Joosten, R. (1996), Dynamics, Equilibria and Values, Dissertation, Maastricht University.

Ju, Y., P.E.M. Borm, and P.H.M. Ruys (2007) "The Consensus Value : a New Solution Concept for Cooperative Games", Social Choice and Welfare, 28, 685-703.

Ju, Y., and D. Wettstein (2009) "Implementing Cooperative Solution Concepts: a Generalized Bidding Approach", Economic Theory, 39, 307-330.

Maschler M, and G.Owen (1989) "The Consistent Shapley Value for Hyperplane Games", International Journal of Game Theory, 18, 389407.

Myerson, R.B. (1980) "Conference Structures anf Fair Allocation Rules", International Journal of Game Theory, 9, 169-182.

Nash, J.F. (1953) "Two-Person Cooperative Games" Econometrica, 21: 128-140.

Pérez-Castrillo, D. and D. Wettstein (2001) "Bidding for the Surplus: a Non-Cooperative Approach to the Shapley Value", Journal of Economic Theory 100, 274-294.

Shapley, L.S. (1953) "A Value for n-Person Games", in: Annals of Mathematics Studies 28 (Contributions to the Theory of Games Vol.2) (eds. H.W. Kuhn and A.W. Tucker), Princeton University Press, pp.307-317. 
Sobolev, A.I (1973) "The Functional Equations that Give the Payoffs of the Players in an N-Person Game", in: Advaces in Game Theory (ed. E. Vilkas), Izdat. "Mintis", Vilnius, pp.151-153 (in Russion).

Young, H.P. (1985), "Monotonic Solutions of Cooperative Games", International Journal of Game Theory, 14, 65-72. 